Jason Russ, MD

Assistant Professor of Clinical Medicine,

Indiana University School of Medicine, Indiana

University Health Physicians, Indianapolis, IN
Areeba Kara, MD, MS, FACP, SFHM

Assistant Professor of Clinical Medicine,

Indiana University School of Medicine, Indiana

University Health Physicians, Indianapolis, IN

\title{
Gastroenteritis gone rogue
}

\begin{abstract}
56-year-old woman presented to the A emergency department with a 2-day history of vomiting. She reported vomiting almost immediately after eating and described her emesis as nonbilious and nonbloody. These symptoms were associated with mild, central, intermittent abdominal pain, generalized weakness, and nonbloody, nonmucoid diarrhea. She described 5 episodes of watery stools per day. She said she had no fever, recent travel, previous similar episodes, or sick contacts. Her symptoms were thought to be consistent with infectious gastroenteritis.

The patient had a history of hypertension and had undergone a partial hysterectomy for uterine fibroids. Her current medications included oral hydrochlorothiazide $25 \mathrm{mg}$ daily and over-the-counter ibuprofen and acetaminophen occasionally for back pain. She worked as a medical assistant at a local hospital. She did not consume alcohol or use illicit drugs, and she smoked 5 cigarettes per day.
\end{abstract}

\section{CHRONIC OR ACUTE?}

1 What is the duration of symptoms consistent with the definition of "acute" diarrhea?

Less than 2 days

Less than 7 days

Less than 14 days

Less than 4 weeks

Diarrhea is defined as acute when it lasts less than 14 days. ${ }^{1}$ This duration allows the resolution of most self-limited gastrointestinal illnesses. Conversely, diarrhea lasting more than 4 weeks is considered chronic and requires investigation. ${ }^{1}$ Our patient presented with acute diarrhea, ie, with symptoms lasting 2 days.

doi:10.3949/ccjm.87a.19007

\section{CASE CONTINUED: EVALUATION IN THE EMERGENCY DEPARTMENT}

In the emergency department, the patient appeared comfortable. Her heart rate was 111 beats per minute, blood pressure 107/65 mm $\mathrm{Hg}$, respiratory rate 20 breaths per minute, and oxygen saturation $97 \%$ on room air. Orthostatic vital signs were not checked, but she was ambulating without symptoms. She was afebrile, with a temperature of $37^{\circ} \mathrm{C}\left(98.6^{\circ} \mathrm{F}\right)$, and she was alert and oriented.

Her oral mucosa was dry. No heart murmurs were heard. Her abdomen was soft, nontender, and nondistended, with normal bowel sounds. Her chest was clear to auscultation. There was no palpable organomegaly. No rash was noted. Neurologic examination was normal. No lymphadenopathy was noted.

Initial laboratory blood results revealed the following values:

- Sodium 134 mmol/L (reference range 136-144)

- Blood urea nitrogen $58 \mathrm{mg} / \mathrm{dL}$ (7-21)

- Creatinine $2.70 \mathrm{mg} / \mathrm{dL}(0.58-0.96)$

- Aspartate aminotransferase (AST) 20 U/L (13-35)

- Alanine aminotransferase (ALT) $24 \mathrm{U} / \mathrm{L}$ (7-38)

- Hemoglobin $12.2 \mathrm{~g} / \mathrm{dL}(11.5-15.5)$

- Platelet count $221 \times 10^{9} / \mathrm{L}(150-400)$

- White blood cell count $9.4 \times 10^{9} / \mathrm{L}$ (3.711.0).

Urinalysis showed the following values:

- Protein $30 \mathrm{mg} / \mathrm{dL}$

- Ketones 5 mg/dL

- Leukocyte esterase $25 \mathrm{mg} / \mathrm{dL}$. Urine microscopy showed the following:

- White blood cells 5-10 per high-power field

- Trace bacteria

- Few squamous epithelial cells

- More than 20 hyaline casts.
A woman presented with 2 days of vomiting and diarrhea 
Noncontrast computed tomography of the abdomen and pelvis showed no abnormally dilated or distended loops of bowel or other pathology.

\section{ARE STOOL STUDIES NEEDED?}

2 Which of the following characteristics of this patient's history and presentation warrant further diagnostic workup with stool studies?

Age $>50$

Employment as a healthcare worker

Evidence of volume depletion

Both healthcare employment and volume depletion

Diagnostic stool studies are indicated in acute diarrhea when there are public health concerns (eg, the patient works in a hospital, in daycare, or as a food-handler) and when the clinical presentation indicates significant volume depletion, both of which were present in this patient. ${ }^{2}$

Our patient's employment as a healthcare worker increases the potential of public health consequences of infectious diarrhea by exposing vulnerable populations. Additionally, the initial examination indicated volume depletion with tachycardia and normotension (rather than hypertension) in a patient who carries the diagnosis of hypertension. Laboratory testing also revealed acute kidney injury, with a blood urea nitrogen-to-creatinine ratio greater than 20:1, and hyaline casts in the urine, suggesting volume depletion.

Other features that warrant stool testing (but were absent in this case) include fever, bloody diarrhea, severe abdominal pain, sepsis, age over 70, immune-compromised status, known or suspected inflammatory bowel disease, community outbreaks, pregnancy, and significant comorbidities. ${ }^{1-3}$

\section{CASE CONTINUED:} SHE IS ADMITTED AND SPIKES A FEVER

The patient was admitted and started on intravenous normal saline at $100 \mathrm{~mL} /$ hour. Over the next 12 hours her symptoms resolved and her creatinine improved to $1.4 \mathrm{mg} / \mathrm{dL}$. However, the next day she developed a fever, with a temperature reaching $39.2^{\circ} \mathrm{C}\left(102.6^{\circ} \mathrm{F}\right)$. Stool studies for gastrointestinal pathogens including Salmonella sp., Shigella sp., Escherichia coli, and Clostridioides difficile returned negative.

The patient continued to be febrile with sustained daily temperatures of $39^{\circ} \mathrm{C}$. She reported a cough productive of thick white sputum. Intermittent tachycardia with a heart rate up to 120 beats per minute was noted.

Chest radiography showed increased interstitial markings. Auscultation of the lungs revealed bilateral crackles. Noncontrast computed tomography of the chest revealed bilateral ground-glass opacities. A ventilationperfusion scan was negative for pulmonary embolism.

A nasopharyngeal swab was sent for viral pathogens and blood cultures.

\section{ELEVATED PROCALCITONIN}

Procalcitonin was measured and found to be elevated at $58.02 \mathrm{ng} / \mathrm{mL}$. Reference levels for procalcitonin are as follows:

- $\leq 0.5$ - sepsis is not likely

- $0.5-2.0$ - sepsis is possible

- > 2.0—sepsis is likely, unless other causes are known

- $\geq 10.0$-important systemic inflammatory response, almost exclusively due to severe bacterial sepsis or septic shock.

3 This procalcitonin level supports a diag3 nosis of lower respiratory tract infection with which of the following pathogens?

Typical bacteria

Atypical bacteria

A virus

Fungi

Typical bacteria are most likely. In adults hospitalized with community-acquired pneumonia, procalcitonin levels were noted by Self et al to be highest in those with typical bacterial infection. ${ }^{4}$ Patients with viral or fungal pneumonia had lower procalcitonin elevations than those with atypical or typical bacterial infections; patients with a procalcitonin level of $10 \mathrm{ng} / \mathrm{mL}$ were 4 times more likely to have a bacterial pathogen than those with an undetectable procalcitonin $(<$ $0.05 \mathrm{ng} / \mathrm{mL}){ }^{4}$

The marked procalcitonin elevation in 
this patient exceeds the thresholds typically described (which range from 0.25 to $1 \mathrm{ng} / \mathrm{mL}$ ) and may reflect bacterial illness or nonbacterial severe systemic inflammation. ${ }^{5}$

\section{CASE CONTINUED:} HER CONDITION WORSENS

Treatment was started with azithromycin, vancomycin, and piperacillin-tazobactam to cover atypical bacterial pathogens that may cause hospital-acquired pneumonia, but fevers continued.

On hospital day 5 the patient developed atrial fibrillation with rapid ventricular response requiring transfer to the progressive care unit. Intravenous diltiazem was administered, resulting in conversion to normal sinus rhythm.

Further laboratory testing indicated worsening leukocytosis, with white blood cell counts up to $18.9 \times 10^{9} / \mathrm{L}$ and worsening renal function after her initial recovery. New thrombocytopenia developed, with platelet counts falling to $109 \times 10^{9} / \mathrm{L}$. She was now requiring supplementary oxygen by nasal cannula at 3 to $4 \mathrm{~L}$ per minute to maintain adequate saturation.

\section{NEW ATRIAL FIBRILLATION IN SEPSIS}

1 What is the incidence of new-onset atrial fibrillation in patients with severe sepsis?

$\square 2 \%$
$\square 5 \%$
$\square 10 \%$
$\square 15 \%$

The incidence of new-onset atrial fibrillation in patients with severe sepsis is $10 \%$.

Cardiac arrhythmias are common in patients with sepsis, and atrial fibrillation is the most common. Physiologic stressors with resulting autonomic surges, volume shifts, and stress hormones are all contributing factors. ${ }^{6}$ The incidence increases with illness severity, and the incidence of new-onset atrial fibrillation in patients with septic shock is estimated at $23 \% .{ }^{6} \mathrm{New}$-onset atrial fibrillation is associated with longer lengths of stay and increased risk of in-hospital death.?

\section{CASE CONTINUED:} TRANSFER TO INTENSIVE CARE

The patient's fevers continued, and her renal function, bilirubin, and thrombocytopenia continued to worsen. The creatinine level increased to $3.36 \mathrm{mg} / \mathrm{dL}$. Evidence of new hepatic inflammation was noted, with the following blood values:

- Total bilirubin $22.3 \mathrm{mg} / \mathrm{dL}$

- Direct bilirubin $18.4 \mathrm{mg} / \mathrm{dL}$

- ALT $192 \mathrm{U} / \mathrm{L}$

- AST $174 \mathrm{U} / \mathrm{L}$

- Lactate dehydrogenase (LDH) 290 U/L

- Platelet count $49 \times 10^{9} / \mathrm{L}$

- Hemoglobin $7.3 \mathrm{~g} / \mathrm{dL}$

- White blood cell count $32.9 \times 10^{9} / \mathrm{L}$, with $2 \%$ myelocytes, $3 \%$ bands, $92 \%$ neutrophils, $2 \%$ lymphocytes, and $1 \%$ monocytes

- Free thyroxine $0.8 \mathrm{ng} / \mathrm{dL}$

- Fibrinogen $740 \mathrm{mg} / \mathrm{dL}$

- International normalized ratio 1.03

- Ferritin 2,455 ng/mL.

Peripheral smear results showed nucleated red blood cells, leukocytosis with left shift, and thrombocytopenia. Blood, urine, and sputum cultures showed no growth. A respiratory viral panel was also negative.

On hospital day 7, the patient was transferred to the intensive care unit due to worsening mentation, persistent fevers, deteriorating renal function, shock, and respiratory distress requiring continuous venovenous hemofiltration, pressors, and mechanical ventilation. No rash or lymphadenopathy developed in the interim.

Progressive thrombocytopenia led to the consideration of acute disseminated intravascular coagulation (DIC).

\section{IS THIS ACUTE DISSEMINATED INTRAVASCULAR COAGULATION?}

5 Which of the following laboratory profiles is expected in acute DIC?

Elevated fibrinogen, prolonged prothrombin time, and reduced platelet count

Reduced fibrinogen, prolonged prothrombin time, and reduced platelet count
Her

procalcitonin was elevated at $58.02 \mathrm{ng} / \mathrm{mL}$ 
Elevated fibrinogen, prolonged prothrombin time, and elevated D-dimer

In DIC, the normal processes of coagulation and fibrinolysis become dysregulated and abnormally activated within the vasculature. Causes include sepsis, malignancy, trauma, and obstetric complications. It is a clinicopathologic diagnosis requiring both consideration of the clinical context and interpretation of laboratory data.

In acute DIC, the fibrinogen level is typically low with prolongation of the prothrombin and activated partial thromboplastin times accompanying thrombocytopenia (the second answer choice above). ${ }^{8}$ Thus, this patient's laboratory values are not consistent with acute DIC, as she had a normal international normalized ratio and elevated fibrinogen.

Nucleated red blood cells in the peripheral smear may indicate extreme stress, hemolysis, extramedullary hematopoiesis, hypoxia, or pathology that displaces normal bone marrow (myelophthisis), ${ }^{8}$ and they predict a higher risk of death. ${ }^{9}$

\section{She was diagnosed with hemo- phagocytic lymphohistio- cytosis}

\section{CASE CONTINUED:} THE DIAGNOSIS RECONSIDERED

The patient became critically ill, with multiorgan system failure. The negative cultures argued against active bacterial infection. Noninfectious causes were systematically considered. Acute DIC did not explain her thrombocytopenia, and with ongoing fevers and nucleated red blood cells in the periphery, the bone marrow was considered as a site of pathology. Disorders of the myeloid system that result in activation of the inflammatory cascade include leukemia. With multiple organs now affected, new hepatic injury, and elevated ferritin levels, hemophagocytic lymphohistiocytosis (HLH) was considered.

\section{HEMOPHAGOCYTIC LYMPHOHISTIOCYTOSIS}

6 Which additional laboratory values should be obtained to support the diagnosis of $\mathrm{HLH}$ ?

Fasting triglycerides

Total cholesterol

Repeat procalcitonin

Serologic testing for Epstein-Barr virus
HLH is a syndrome characterized by excessive immune activation. According to the 2004 Histiocyte Society guidelines, ${ }^{10}$ the diagnosis of HLH requires 5 of the following 8 criteria in the absence of evidence of malignancy:

- Fever

- Splenomegaly

- Cytopenias affecting at least 2 cell lines: hemoglobin $<90 \mathrm{~g} / \mathrm{L}(<100 \mathrm{~g} / \mathrm{L}$ in infants $<4$ weeks old), platelet count $<100 \times 10^{9} / \mathrm{L}$, or neutrophil count $<1.0 \times 10^{9} / \mathrm{L}$

- Fasting triglycerides $\geq 265 \mathrm{mg} / \mathrm{dL}$ or fibrinogen $\leq 1.5 \mathrm{~g} / \mathrm{L}$, or both

- Hemophagocytosis in the bone marrow, spleen, or lymph nodes

- Low or absent natural killer (NK) cell activity

- Ferritin $\geq 500 \mathrm{ng} / \mathrm{mL}$

- CD25 (soluble interleukin 2 receptor) $\geq 2,400 \mathrm{U} / \mathrm{mL}$.

Alternatively, the patient should have a molecular diagnosis of HLH based on the detection of genetic mutations related to the pathogenesis of HLH.

\section{CASE CONTINUED: FURTHER TESTING}

Further testing showed the following:

- Fasting triglycerides $316 \mathrm{mg} / \mathrm{dL}$ (reference range $<150$ )

- CD25 2,532 U/mL (45-1,105)

- Normal NK cell function

- No mutations linked to HLH detected on genetic testing: AP3B1, BLOC156, LYST, RAB27A, SH2D1A, SLC7A7, STX11, and XIAP were normal

- Hemophagocytosis on bone marrow biopsy study, consistent with HLH.

This patient met 6 of the 8 HLH diagnostic criteria, ie, fever, bicytopenia (anemia and thrombocytopenia), hypertriglyceridemia, hemophagocytosis in the bone marrow, elevated ferritin, and increased soluble CD25.

In clinically deteriorating patients, treatment for HLH should not be initiated until which of the following tests is completed?

Demonstration of hemophagocytosis on bone marrow evaluation Molecular testing for genetic mutations Neither of the above 
Neither of the above.

Delayed treatment portends a worse prognosis. In a single-center study of adult patients with HLH, $66 \%$ of patients had died after a median follow-up of 42 months. The median overall survival of the entire cohort was 2.1 months. ${ }^{11}$

The HLH 2004 diagnostic criteria ${ }^{10}$ are largely based on pediatric data, and presentations in adults may differ. ${ }^{12}$ Demonstration of hemophagocytosis is not required for diagnosis. In an unstable patient in whom the clinical index of suspicion is high, referral to a hematology service and treatment should not be delayed.

\section{CASE CONTINUED:} TREATMENT AND RECOVERY

The diagnosis of HLH was confirmed, and treatment was started with etoposide and dexamethasone, according to the HLH-94 treatment protocol. ${ }^{13}$ After a prolonged hospitalization of 57 days, the patient was discharged home. Over the next 10 months, she had no recurrence.

\section{HEMOPHAGOCYTIC LYMPHOHISTIOCYTOSIS IS LIFE-THREATENING}

HLH is a life-threatening clinical syndrome resulting from an excessive, uncontrolled immune response. It is characterized as primary when it is caused by genetic mutations, or secondary when there are no associated genetic defects. ${ }^{14}$

The implicated genes code proteins responsible for cytotoxic processes. When this pathway is impaired, activated macrophages engulf normal tissues and trigger severe unregulated inflammation. ${ }^{15}$ Primary HLH most commonly presents in children. Secondary HLH may be associated with predisposing conditions causing immune dysregulation, such as lymphoma, immunodeficiency, or autoimmune diseases.

Episodes in either primary or secondary HLH may be triggered by infection, most often with Epstein-Barr virus. ${ }^{16}$

Data on the incidence of HLH in adults are limited, but a nationwide survey in Japan estimated an annual incidence of 1 per $800,000 .^{16}$
Cardinal symptoms include prolonged fever, hepatosplenomegaly, and cytopenias. As noted, the diagnostic guidelines for HLH include a list of 8 clinical and laboratory criteria, 5 of which must be met for the diagnosis of $\mathrm{HLH}$, or the patient should have genetic testing consistent with HLH. ${ }^{10}$

However, HLH criteria are not fulfilled in all cases, and additional manifestations commonly seen in adults are not included in the criteria (eg, elevated AST and LDH). ${ }^{12}$ Fever is noted in almost all presentations at diagnosis, while hepatosplenomegaly and cytopenias are noted in $80 \%$ of patients. Ferritin is elevated in $95 \%$ of cases of secondary HLH, while low fibrinogen is less common at $40 \% .{ }^{14}$ The incidence of skin manifestations ranges from $6 \%$ to $65 \%$, and more than a third of patients have neurologic symptoms ranging from seizures to encephalopathy.

Each of the cardinal clinical and laboratory findings is linked to the underlying pathophysiology. High levels of inflammatory cytokines result in fever. Splenomegaly results from the infiltration by lymphocytes and macrophages. Cytopenias are related to high levels of tumor necrosis factor alpha and interferon gamma and to hemophagocytosis. Elevated triglycerides are caused by the decreased lipoprotein lipase activity initiated by increased tumor necrosis factor alpha levels. Ferritin is believed to accumulate as macrophages scavenge heme. High concentrations of soluble interleukin 2 receptor are produced by activated lymphocytes. ${ }^{14}$ Liver function derangements are related to direct invasion of the liver and biliary tree by activated macrophages with resulting marked elevations of bilirubin and elevations in AST, ALT, and LDH. ${ }^{13}$

Hemophagocytosis is not required for the diagnosis of HLH and it neither confirms nor excludes the diagnosis. Fewer than half of patients may have hemophagocytosis evident on initial presentation. ${ }^{17}$

Differentiating between sepsis and HLH may be difficult due to the rarity of the disease in the adult population, but if suspicion is high, the pattern of clinical findings and disease progression can be used to aid the diagnosis. ${ }^{18} \mathrm{Se}$ rial markers of inflammation, ferritin, and bone marrow evaluation may also aid the diagnosis.

The death rate in adult HLH has been re-
She was sent home after 57 days, and over the next 10 months had no recurrence 
ported to range from $20 \%$ to $88 \% .{ }^{16}$ Every patient with suspected HLH should be referred urgently to a hematologist, as prompt initiation of treatment is important to improve survival. Treatment does not need to wait for definite diagnosis if suspicion is high. The initial goal of treatment is to suppress the excessive life-threatening inflammatory cascade; most regimens follow the HLH-94 protocol, which includes steroids and etoposide. After 8 weeks, patients are either weaned off therapy or transitioned to continuation therapy. ${ }^{14}$

\section{KEY POINTS}

- HLH is primarily a disease of children but can be seen in adults.

- Hemophagocytosis is not always evident and is not required for the diagnosis of $\mathrm{HLH}$.

- Management requires input from specialists, and treatment should not be delayed for molecular diagnosis.

- HLH may be triggered by an illness, with Epstein-Barr virus being the most common infectious agent associated with it.

\section{REFERENCES}

1. Sweetser S. Evaluating the patient with diarrhea: a case-based approach. Mayo Clin Proc 2012; 87(6):596-602. doi:10.1016/j.mayocp.2012.02.015

2. Riddle MS, DuPont HL, Connor BA. ACG clinical guideline: diagnosis, treatment, and prevention of acute diarrheal infections in adults. Am J Gastroenterol 2016; 111(5):602-622. doi:10.1038/ajg.2016.126

3. Shane AL, Mody RK, Crump JA, et al. 2017 Infectious Diseases Society of America clinical practice guidelines for the diagnosis and management of infectious diarrhea. Clin Infect Dis 2017; 65(12):e45e80. doi:10.1093/cid/cix669

4. Self WH, Balk RA, Grijalva CG, et al. Procalcitonin as a marker of etiology in adults hospitalized with community-acquired pneumonia. Clin Infect Dis 2017; 65(2):183-190. doi:0.1093/cid/cix317

5. Fakheri R. Can procalcitonin guide decisions about antibiotic management? Cleve Clin J Med 2019; 86(5):307-311. doi:10.3949/ccjm.86a.18074

6. Kuipers S, Klouwenberg PM, Cremer OL. Incidence, risk factors and outcomes of new-onset atrial fibrillation in patients with sepsis: a systematic review. Crit Care 2014; 18(6):688. doi:10.1186/s13054-014-0688-5

7. Moss TJ, Calland JF, Enfield KB, et al. New-onset atrial fibrillation in the critically ill. Crit Care Med 2017; 45(5):790-797. doi:10.1097/CCM.0000000000002325

8. Jameson J, Fauci AS, Kasper DL, Hauser SL, Longo DL, Loscalzo J. eds. Harrison's Principles of Internal Medicine, 20th ed. New York, NY: McGraw-Hill; 2018.

9. Stachon A, Sondermann N, Imohl M, Krieg M. Nucleated red blood cells indicate high risk of in-hospital mortality. J Lab Clin Med 2002; 140(6):407-412. doi:10.1067/mlc.2002.129337

10. Henter JI, Horne A, Aricó M, et al. HLH-2004: Diagnostic and therapeutic guidelines for hemophagocytic lymphohistiocytosis. Pediatr
Blood Cancer 2007; 48(2):124-131. doi:10.1002/pbc.21039

11. Parikh SA, Kapoor P, Letendre L, Kumar S, Wolanskyj AP. Prognostic factors and outcomes of adults with hemophagocytic lymphohistiocytosis. Mayo Clin Proc 2014; 89(4):484-492. doi:10.1016/j.mayocp.2013.12.012

12. Hejblum G, Lambotte $\mathbf{O}$, Galicier $\mathbf{L}$, et al. A web-based delphi study for eliciting helpful criteria in the positive diagnosis of hemophagocytic syndrome in adult patients. PLoS One 2014; 9(4):e94024. doi:10.1371/journal.pone.0094024

13. Henter J-I, Arico M, Egeler M, et al. HLH-94: a treatment protocol for hemophagocytic lymphohistiocytosis. Med Pediatr Oncol 1997; 28(5):342-347. doi:10.1002/(sici)1096-911x(199705)28:5<342::aid-mpo3>3.0.co;2-h

14. George MR. Hemophagocytic lymphohistiocytosis: review of etiologies and management. J Blood Med 2014; 5:69-86. doi:10.2147/JBM.S46255

15. Jordan MB, Allen CE, Weitzman S, Filipovich AH, McClain KL. How I treat hemophagocytic lymphohistiocytosis. Blood 2011; 118(15):4041-4052. doi:10.1182/blood-2011-03-278127

16. Hayden A, Park S, Giustini D, Lee AY, Chen LY. Hemophagocytic syndromes (HPSs) including hemophagocytic lymphohistiocytosis (HLH) in adults: a systematic scoping review. Blood Rev 2016; 30(6):411420. doi:10.1016/j.blre.2016.05.001

17. Janka GE. Familial and acquired hemophagocytic lymphohistiocytosis. Annu Rev Med 2012; 63:233-246. doi:10.1146/annurev-med-041610-134208

18. Machowicz R, Janka G, Wiktor-Jedrzejczak W. Similar but not the same: differential diagnosis of HLH and sepsis. Crit Rev Oncol Hematol 2017; 114:1-12. doi:10.1016/j.critrevonc.2017.03.023

ADDRESS: Jason Russ, MD, Indiana University School of Medicine, $1800 \mathrm{~N}$ Capitol Avenue, Suite E140, Indianapolis, IN 46202; jruss1@iuhealth.org 